\title{
A Novel Representation of Energy and Signal Transformation in Measurement Systems
}

\author{
Qingping Yang, Member, IEEE
}

\begin{abstract}
This paper presents a novel representation of energy and signal transformation in a measurement system, which is essentially a transducer conversion logic or language (TCL). Using two-port and three-port transducers as basic building blocks, it can be utilized to model any measurement system. It has the key features of object-orientation and consists of only text with very simple syntax. The TCL can be easily handled and processed by computers. This paper has demonstrated its use in description, classification, and computer-aided analysis and design of measuring instruments with some preliminary test results. It will find wide applications in modeling, analysis, design, and education in measurement, control, and information processing.
\end{abstract}

Index Terms-Measurement science, modeling, object-oriented methods, system analysis and design, virtual instrumentation.

\section{INTRODUCTION}

$\mathbf{T}$ HE INFORMATION in a measurement system is carried and conveyed by signals, which in turn, reside in a certain power or energy form. Signals can be regarded as the factors of energy or power.

A transducer has energy connections with some other elements and the environment. Each connection interface is often called the power port. Intrinsically (without considering environmental interactions), a transducer has two or three power ports, i.e., input, output, and modulation (or sometimes called interrogation), although modulation is not always necessary. A two-port transducer is also called a self-generating or passive transducer (e.g., piezoelectric transducer), and a three-port transducer called a modulating or active transducer (e.g., strain gauge) [1], [2].

The input energy, output energy, and, if necessary, the modulation energy together define the transformation (or conversion mechanism or transduction principle) of a transducer.

The representation of energy and signal transformation in a measurement process plays an important role in the modeling, design, and analysis of a measuring instrument. The traditional approach is based upon the use of a block diagram, signal flow diagram, and bond graph [3]. More recently, three-dimensional (3-D) and matrix representations have been proposed [2], [4]. They are based upon the classification of transducers and physical effects according to their energy forms, i.e., electrical, mechanical, thermal, radiant, magnetic, and chemical, six signal domains.
In the 3-D representation, the energy forms of input, output, and modulation correspond to three axes (i.e., $x, y$ and $z$ axes, respectively), and each conversion principle or physical effect can thus be shown as a "sensor effect cube" (i.e., a cube region in a 3-D coordinate system) [2]. Consequently, each element of a measuring chain may be expressed as 3-D coordinates, e.g., $[x, y$, and $z]$, where $x, y$ and $z$ are one of the six energy forms. Based upon the 3-D representation, Middlehoek and Noorlag [2] have further proposed a methodology for describing a measuring instrument by joining the 3-D coordinates of each stage, with additional lines drawn to show the energy source/flow as well as the signal flow. Although it is an interesting idea, it is rather limited because only the energy form is given. It does not seem to be natural to use, and it is quite difficult to interpret a conversion process.

The matrix representation shows each transducer as a transformation column, with the left and right vertical edges corresponding to the input and output energy form, respectively [2], [3]. A line may be drawn to connect the input and output domains, representing the transformation from one energy form to another. All the elements of a measuring chain can be represented as such columns and connected together as a matrix. The conversion lines may be joined together in the transformation matrix of a measuring chain [2].

It is quite straightforward to use this kind of matrix representation to describe conversion processes and energy flows. However, it does not include the modulation input directly, and the representations of energy flow and signal flow are not integrated.

Both the 3-D and matrix approaches proposed are mainly graphic presentations, and, as such, it is neither easy nor efficient to handle these representations using computers.

This paper presents a novel representation of energy and signal transformation in measurement systems.

\section{PRINCIPLE AND PRoposed APPROACH}

The proposed representation is based upon both energy and signal flows. It has a natural link to the signal flow diagram, block diagram, and bond graph. It may be generally referred to as transducer conversion logic or language (TCL).

According to this approach, a two-port (or self-generating) and three-port (or modulating) transducer or element can be represented as

$$
x \rightarrow y
$$

and

Manuscript received June 15, 2003; revised May 27, 2004.
The author is with the Department of Systems Engineering, Brunel University, Middlesex UB8 3PH, U.K. (e-mail: emstppy@brunel.ac.uk).

Digital Object Identifier 10.1109/TIM.2004.834611

$$
x \mid z \rightarrow y
$$


respectively, where $x, y$, and $z$ are input, output, and modulation of a transducer, respectively. The arrow represents a functional relationship between output and input variables (including the modulation). Each variable takes a form of D.s, where $s$ is the signal concerned, and $D$ is one of the six energy forms (or signal domains), which may be identified by the following initials: $E$, Electrical; $M e$, Mechanical; $T$, Thermal; $R$, Radiant; $M a$, Magnetic; and $C$, Chemical.

For example, a piezoelectric transducer has a direct conversion process

$$
M e . F \rightarrow E . q
$$

which converts mechanical force $(F)$ into electrical charge $(q)$. A strain gauge connected to a bridge circuit can be represented as

$$
M e . \varepsilon \rightarrow E . R \mid E . S_{v} \rightarrow E . v
$$

where the strain $(\varepsilon)$ is converted to electrical resistance $(R)$, which is then converted to a voltage output $(v)$ with voltage modulation, i.e., voltage source $\left(S_{v}\right)$.

Of course, longer names can also be used for signals, if necessary, but as the signal domain is specified, it is quite easy to understand standard symbols for variables or signals which are commonly used. In fact, the same symbol, but in different domains, can be easily resolved. This makes the representation more powerful.

The above conversion expression for a three-port or modulating transducer can be expanded to model a more complicated process in which the modulation itself is converted from another source

$$
x \mid z \rightarrow y
$$

where $z=x 1 \rightarrow z 1$ or $z=x 1 \mid y 1 \rightarrow z 1$.

The combined conversion can be expressed as

$$
x \mid(z \leftarrow x 1) \rightarrow y
$$

or

$$
x \mid(z \leftarrow x 1 \mid z 1) \rightarrow y
$$

Take, for example, the above strain gauge connected to a bridge circuit, and assume the voltage supply is converted from an optical light source which, in turn, is controlled by an electric current source. Then, the process can be expanded as

$$
M e . \varepsilon \rightarrow E . R \mid\left(E . S_{v} \leftarrow R . I \mid E . S_{i}\right) \rightarrow E . v
$$

where $R . I$ is the light intensity in radiant $(R)$ domain, $E . S_{v}$ is the voltage source, and E.S $S_{i}$ is the current source.

\section{RESUlTS AND DiscusSiON}

As a modeling language, the proposed TCL can be used to model the flow of energy, signal, and information in the system concerned. A number of applications in instrumentation and measurement are demonstrated in this section, including a

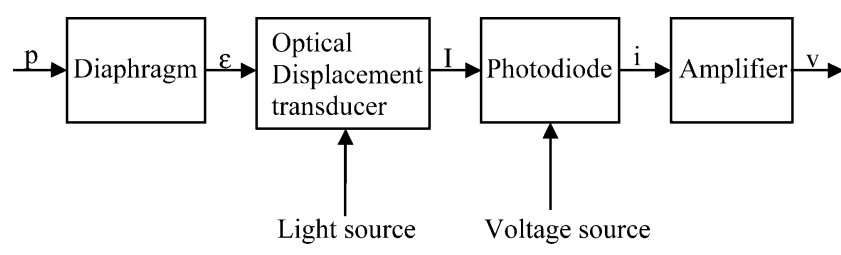

Fig. 1. Block diagram of the pressure transducer.

description of measuring instruments, classification, computeraided analysis, and design of measuring instruments. Some of these have been tested, and preliminary test results are also presented.

\section{A. Natural Description of Measuring Instruments}

This may be illustrated by the example of a pressure transducer. Assume the measured pressure is applied to a mechanical diaphragm, whose deformation is sensed by an intensity-modulated optical fiber sensor. The light intensity is then converted to electrical current by a photodiode and finally converted to a voltage with a transimpedance amplifier. The measuring chain may be first represented as

$$
M e . p \rightarrow M e . \varepsilon \rightarrow R . I \rightarrow E . i \rightarrow E . v
$$

where $p$ is measured pressure, $\varepsilon$ is diaphragm deformation, $I$ is the output light intensity from the optical fiber sensor, $i$ is the current output from the photodiode, and $v$ is the amplifier voltage output.

More details about the light intensity modulation can be added as follows

$$
M e . \varepsilon \mid R . S_{I} \rightarrow R . I
$$

where $S_{I}$ is the light source, and

$$
M e . \varepsilon \mid\left(R . S_{I} \leftarrow E . S_{i}\right) \rightarrow R . I
$$

where $S_{i}$ is the current source.

The latter expression shows the mechanical deformation is converted to light intensity, with the light source powered by an electric current source. Note that more details can be simply added without affecting the structure.

Assuming the photo detector is voltage biased, then we have

$$
R . I \mid(E . v=\text { constant }) \rightarrow E . i .
$$

The expanded chain can thus be expressed as

$M e . p \rightarrow M e . \varepsilon\left|\left(R . S_{I} \leftarrow E . S i\right) \rightarrow R . I\right| E . S v \rightarrow E . i \rightarrow E . v$.

In fact, this expression may be easily converted to a signal flow diagram or a block diagram, as shown in Fig. 1.

\section{B. Classification of Measuring Instruments}

The TCL can be readily used to classify various physical transducer principles. For example, a conventional strain gauge is classified as $M e . \varepsilon \rightarrow E . R$, and a bridge circuit is $E . R \mid E . S_{v} \rightarrow E . v$. This could be further condensed as a vector or coordinates, i.e., $(M e . \varepsilon ; E . R),\left(E . R, E . S_{v} ; E . v\right)$. 
If each signal domain is coded, e.g., $E=0, M e=1, T=2$, $R=3, M a=4$, and $C=5$, then the above two transducer principles may be classified as $(1 \varepsilon ; 0 R)$ and $\left(0 R, 0 S_{v} ; 0 v\right)$ or simply $1 \varepsilon 0 R$ and $0 R 0 S_{v} 0 v$. Of course, the signals in each domain can also be coded, e.g., in one decimal digit $\varepsilon=1, R=0$, $S_{v}=2, v=2$. Then we have 1100.000202 to uniquely identify the above two transducer principles. One example application is given in Section III-C on instrumentation design.

\section{Computer-Aided Instrumentation Design}

The TCL or its classification codes can be easily stored for each kind of transducer in a database. Since an instrument is essentially a measuring chain, i.e., a chain of transducers (in the general sense), the essential design task is to search in the database to match TCL or their classification codes.

For example, given a task to design an instrument for measuring mechanical strain, the above two devices will be selected because the strain gauge code 1100 matches the bridge circuit code $\mathbf{0 0 0 2 0 2}$, i.e., the last two digits of the strain gauge code are the same as the first two digits of the bridge circuit code.

The design generated by the computer is generally not unique. For instance, the code 000202 could also be a voltage divider. This means that an alternative design can also be generated, but additional performance analysis and criteria are required to rank the suitability for a given application. The computer-aided analysis of a measuring instrument, as discussed in Section III-D, can also be used in the design.

\section{Computer-Aided Analysis of Measuring Instruments}

A more complicated closed-loop accelerometer is used to demonstrate the computer-aided analysis of measuring instruments, with its block diagram shown in Fig. 2 [5]. The measured acceleration is converted to an inertia force by a seismic mass $(m)$. This force is balanced by the force produced by a feedback element (e.g., through a permanent magnet on a current feedback coil). The unbalanced force is converted to a displacement by an elastic force transducer (e.g., a spring-mass-damper element). The potentiometer displacement transducer converts the displacement to a voltage, which is then amplified, giving a current output to the feedback coil and producing a voltage output through a resistor $(R)$.

Based upon the proposed TCL, the above block diagram can be represented as follows

$$
\begin{aligned}
M e . a & \rightarrow M e . F_{i} \mid M e . F_{B} \rightarrow M e . F_{u} \\
& \rightarrow M e . d \rightarrow E . v \rightarrow E . i \rightarrow E . V_{o}
\end{aligned}
$$

where $M e . F_{B}$ may be regarded as a generalized modulation to Me.F.

The functional relations (or transfer functions) represented by the arrows can be embedded or explicitly described. This can be further expanded as

$$
\begin{gathered}
M e . a \stackrel{f_{1}}{\longrightarrow} M e . F_{i} \mid\left(M e . F_{B} \stackrel{g_{1}}{\longrightarrow} E . i\right) \stackrel{f_{2}}{\longrightarrow} M e . F_{u} \stackrel{f_{3}}{\longrightarrow} \ldots \\
\stackrel{f_{3}}{\longrightarrow} M e . d \mid E . S_{v} \stackrel{f_{4}}{\longrightarrow} E . v \stackrel{f_{5}}{\longrightarrow} E . i \stackrel{f_{6}}{\longrightarrow} E . V_{o} .
\end{gathered}
$$

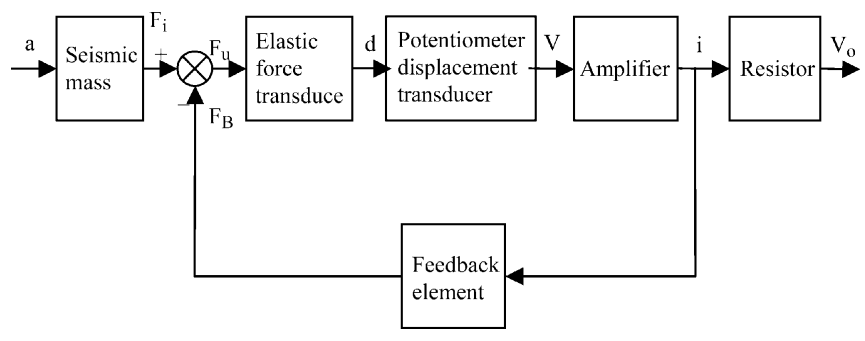

Fig. 2. Block diagram of closed-loop accelerometer.

The embedded transfer functions in this case are as follows:

$$
\begin{aligned}
f_{1}: F_{i} & =m a \\
f_{2}: F_{u} & =F_{i}-F_{B} \\
f_{3}: d & =\frac{\frac{\omega_{n}^{2}}{k}}{s^{2}+2 \xi \omega_{n} s+\omega_{n}^{2}} F_{u} \\
f_{4}: v & =K_{d} d \\
f_{5}: i & =K_{A} v \\
f_{6}: V_{o} & =i R \\
g_{1}: F_{B} & =K_{F} i .
\end{aligned}
$$

The analysis of the instrument can be performed with symbolic math. This was tested in the Maple software, and some preliminarily results were obtained.

1) System Transfer Function: Given the above embedded individual transfer functions for each device, the system transfer function was automatically generated by the Maple as

$$
V_{o}=\frac{K_{A} K_{d} \omega_{n}^{2} m a R}{k s^{2}+2 k \xi \omega_{n} s+k \omega_{n}^{2}+\omega_{n}^{2} K_{f} K_{a} K_{d}} \cdot a
$$

which can then be rearanged as

$$
V_{o}=\frac{m K_{d} K_{A} R}{k+K_{d} K_{A} K_{F}} \cdot \frac{\omega_{n}^{\prime 2}}{s^{2}+2 \xi^{\prime} \omega_{n}^{\prime} s+\omega_{n}^{\prime 2}} \cdot a
$$

where $\omega_{n}^{\prime}=\omega_{n} \sqrt{1+\left(K_{d} K_{A} K_{F} / k\right)}$ and $\xi^{\prime}=$ $\left(\xi / \sqrt{1+\left(K_{d} K_{A} K_{F} / k\right)}\right)$

Of course, the system characteristics and responses, both static and dynamic, can be further determined based upon the system transfer function.

2) Error Analysis and Uncertainty Evaluation: Error analysis and uncertainty evaluation can be performed in two different ways. These have also been tested using Maple symbolic math. The first method is similar to the analysis of the system transfer function. In the above example, the error propagation chain can be easily derived from the above measuring chain (14), i.e.,

$$
\begin{aligned}
M e . \Delta a & \rightarrow \Delta\left(M e . F_{i} \mid M e . F_{B}\right) \rightarrow M e . \Delta F_{u} \\
& \rightarrow M e . \Delta d \rightarrow E . \Delta v \rightarrow E . \Delta i \rightarrow E . \Delta V_{o} .
\end{aligned}
$$

The embedded transfer functions, as shown in (16), were individually differentiated, and each error was solved from the system of derivative equations, resulting in various partial derivatives. The total measurement error was then combined according to the law of error propagation. 
The uncertainty evaluation can be similarly performed since an uncertainty source can be treated mathematically as an error source, and the above partial derivatives are the sensitivity coefficients in the uncertainty evaluation. The total uncertainty is combined according to the law of propagation of uncertainty. However, uncertainty propagation is generally more complicated than error propagation because more sources have to be included, e.g., the uncertainty in material constants and ambient conditions, etc.

The second method for error analysis and uncertainty evaluation derives directly from the system transfer function generated in Section III-D1. The partial derivatives and, hence, sensitivity coefficients are calculated from the system transfer function which was also tested using Maple symbolic math. The total error and uncertainty can be then obtained, similar to the first method.

More results on the computer-aided analysis and design will be published in a separate paper [6].

\section{CONCLUSION}

The TCL presented above has the key features of object-orientation, such as hierarchy and inheritance, piecewise refinement, and information hiding [7]. Thus, it is essentially an object-oriented approach to description and modeling of a measuring chain and conversion processes.

Since the TCL has very simple syntax and consists of only text, it can be easily handled and processed by computers. It is compact, highly efficient, and easy to use. For many transducers, typical conversion mechanisms can be used as default or options, which makes it much easier to automate analysis and design of transducers or instruments.
Although the TCL has been presented in the context of measurement systems, it can be readily applied in other applications, e.g., control and information processing. Indeed, the example shown in Fig. 2 is a typical feedback control system.

The proposed TCL as a fundamental representation scheme of energy and signal transformation will find applications in modeling, analysis, design and education in measurement, control, and information processing. This paper has demonstrated some of these applications with preliminary test results.

\section{REFERENCES}

[1] S. Middlehoek and D. J. W. Noorlag, "Silicon micro-transducers," in Instrument and Technology, B. E. Jones, Ed. Bristol, U.K.: Adam Hilger, 1983, vol. 2, pp. 33-44.

[2] — , "Three-Dimensional representation of input and output transducers," Sens. Actuators, vol. 2, pp. 29-4, 1981.

[3] H. M. Paynter, Analysis and Design of Engineering Systems. Cambridge, MA: MIT Press, 1961.

[4] P. H. Sydenham, N. H. Hancock, and R. Thorn, Introduction to Measurement Science and Engineering. New York: Wiley, 1989.

[5] J. P. Bentley, Principles of Measurement Systems, 2nd ed. White Plains, NY: Longman, 1988.

[6] Q. Yang, Computer Aided Analysis and Design of Measuring Instruments, 2005, submitted for publication.

[7] Q. Yang and C. Butler, "Object-oriented model of measurement systems," IEEE Trans. Instrum. Meas., vol. 47, pp. 104-107, Feb. 1998.

Qingping Yang (M'97) received the diploma degree in instrumentation and measurement from Chengdu Aeronautical Polytechnic, Chengdu, China, in 1983 and the Ph.D. degree from Brunel University, Middlesex, U.K. in 1992.

He was an engineer in the Department of Measurement and Testing, Aircraft Structural Strength Research Institute, Shaanxi Province, China. He is currently a lecturer at Brunel University and his research interests include advanced sensors and transducers, dimensional metrology, measurement science, and information systems. He has published more than 40 papers in these areas. 